\title{
Flexible 16nJ/c.s. 134S/s 6b MIM C-2C ADC using Dual Gate Self-aligned Unipolar Metal-Oxide TFTs
}

\author{
Nikolas Papadopoulos*, Soeren Steudel*, Auke Jisk Kronemeijer ${ }^{\S}$, Marc Ameys*, and Kris Myny* \\ *imec, Large Area Electronics, Heverlee, Belgium \\ ${ }^{\S}$ TNO/Holst Center, Eindhoven, Netherlands
}

\begin{abstract}
In this work a metal-oxide (Indium Gallium Zinc Oxide) 6-bit successive approximation metal-insulator-metal C2C analog to digital converter (ADC) fabricated on $15 \mu \mathrm{m}$ thick flexible substrate is demonstrated. The ADC is operated at a clock speed of $2 \mathrm{kHz}$ and at a power supply of $15 \mathrm{~V}$. The power dissipation including output buffers is $212 \mu \mathrm{W}$ at $15 \mathrm{~V}$ and the a sampling rate of $134 \mathrm{~S} / \mathrm{s}$. The $\mathrm{ADC}$ achieves a figure of merit (FoM) of 16.58nJ/c.s. and no missing codes using dual gate selfaligned n-type thin-film transistors on polymeric substrate.
\end{abstract}

\section{INTRODUCTION}

Analog oriented circuitry on metal-oxide large area technologies (LAE) and flexible substrates using Indium Gallium Zinc Oxide (IGZO) thin-film transistors (TFTs) are being demonstrated in recent literature [1]-[6]. The LAE technology is expanding to other applications than flat panel displays, such as inductive RFID tags [7] for Internet-of-Everything applications, bio-potential measurements [6] and imaging for fingerprint sensing.

More specifically, multiple ADC topology implementations using LAE technologies (a-Si, organic or low-temperature polycrystalline silicon) on glass or flexible substrates have been presented in the literature [8]-[12]. Recently, more specifically $\Delta-\Sigma$ [3] or successive approximation (SAR) implementations [2] are enabling new temperature sensing applications counting on uniformity over large areas, stability and relative high mobility of IGZO TFTs. Other applications, such as in-panel fingerprint sensing on mobile displays, require faster sampling rates than temperature sensing. Etch-stop-layer (ESL) IGZO TFTs exhibit fair stability, very low leakage currents, low fabrication cost and good uniformity at room temperature fabrication but suffer from metal-overlap parasitics. Self-aligned dual gate (SADG) IGZO TFTs can provide faster responses due to negligible overlap parasitics [7]. Therefore, we propose a new design of a 6-bit metal-insulator-metal (MIM) C-2C ADC using unipolar SADG IGZO TFT.

This paper is outlined as follows. The performance and uniformity of the dual gate self-aligned gate metal oxide technology is briefly discussed in section II. The proposed ADC design and operation is presented in section III followed by the experimental results in section V. SectionVI summarizes the $\mathrm{C}-2 \mathrm{C}$ ADC results.

\section{Metal Oxide Technology}

Fig. 1 shows the cross-section of the SADG IGZO technology [13]. The dual-gate transistor comprises of two gates:

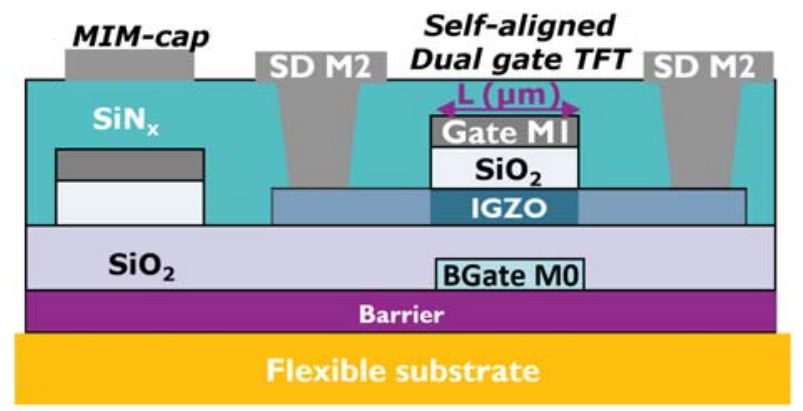

Fig. 1. Dual Gate Self-aligned IGZO TFT and metal-insulator-metal capacitor cross section.

a back-gate (BGate M0) and a front-gate (Gate M1). No additional metals are available for interconnect and routing purposes of the ADC. The self-aligned architecture is selected for its minimum parasitic overlap capacitance between gate and source-drain, its smaller footprint and its ability to decrease the channel length to the critical dimension of the technology, compared to ESL or back-channel etch (BCE) TFT architectures. The total thickness of the wafer is $15 \mu \mathrm{m}$.

SADG TFT were fabricated on GEN1 (320x352mm) substrate size. Since for circuitry performance, 'local' uniformity is key, Fig. 2 shows the transfer curves (IDS-VGS) in saturation of 32 devices across a $150 \mathrm{~mm}^{2}$ wafer area cut down from GEN1, whereby Gate and BGate are connected. The samples have channel width and length of respectively $480 \mu \mathrm{m}$ and $20 \mu \mathrm{m}$, which is the largest footprint TFT used in the design of the ADC. The off current measurement is limited by the semi-automatic measurement setup. Fig. 3 plots the extracted variability on threshold voltage $\left(V_{T}\right)$ and effective dual-gate mobility $\left(\mu_{E F F}\right)$ over $150 \mathrm{~mm}^{2}$ wafer for the 32 devices. The wafer average $V_{T}$ is $1.77 \mathrm{~V}\left(\sigma V_{T}=93 \mathrm{mV}\right)$ and average $\mu_{E F F}$ is $22.97 \mathrm{~cm}^{2} / \mathrm{Vs}\left(\sigma \mu=1 \mathrm{~cm}^{2} / \mathrm{Vs}\right)$ over $150 \mathrm{~mm}^{2}$ area. The apparent mobility is larger compared to literature because of the effect of having two gates controlling the channel.

\section{Details on ADC Design}

Fig. 4 depicts the block diagram of the implemented SAR ADC. The flexible ADC comprises of a C-2C network, a comparator and voltage buffers implemented with the SADG TFT technology. The digital signals (min, min0-6, max0-6, SELIN, res, VAZ) to control the flexible analog TFT chip have been generated by programming a silicon microcontroller. 


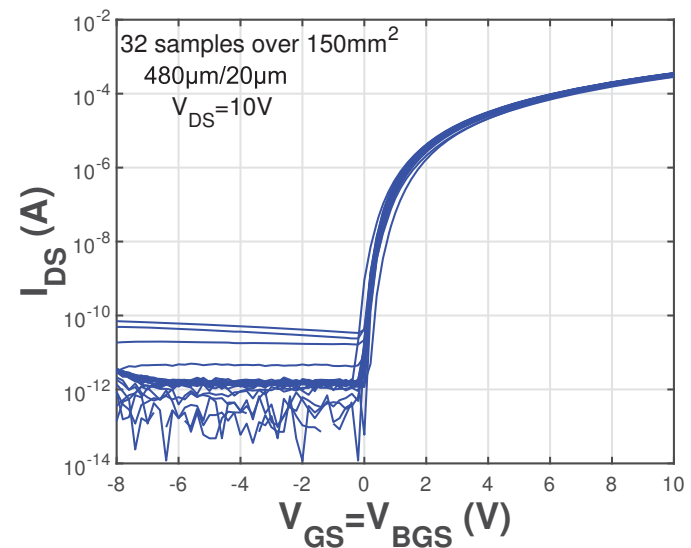

Fig. 2. Measurements of 32 devices of $480 \mu \mathrm{m} / 20 \mu \mathrm{m}$ SADG IGZO TFT across a $150 \mathrm{~mm}^{2}$ wafer.
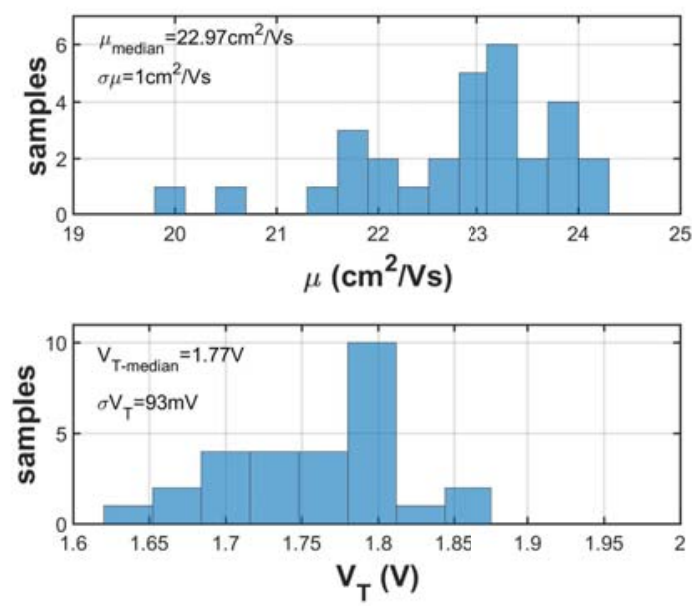

Fig. 3. Wafer threshold voltage $\left(V_{T}\right)$ and effective mobility $\left(\mu_{E F F}\right)$ spread (32 samples) of $\mathrm{W} / \mathrm{L}=480 \mu \mathrm{m} / 20 \mu \mathrm{m}$ SADG IGZO TFTs extracted at $V_{D S}=10 \mathrm{~V}$ across a $150 \mathrm{~mm}^{2}$ wafer.

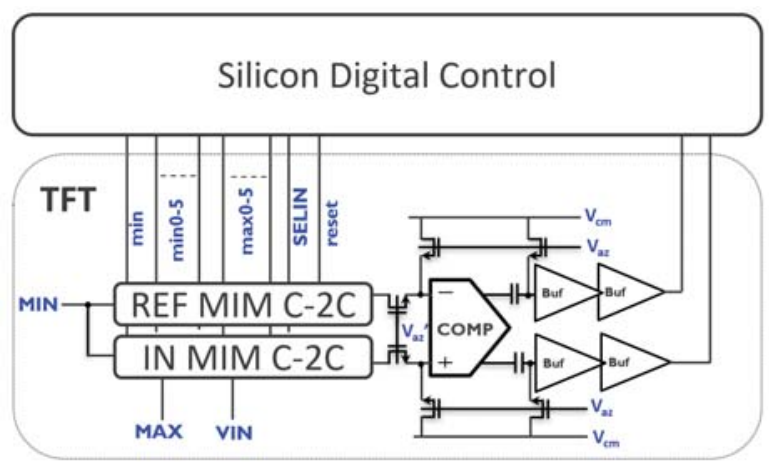

Fig. 4. The implemented SADG TFT ADC block diagram driven with externa silicon digital control.

The C-2C networks are utilizing metal-insulator-metal (MIM) capacitors between Gate and SD layer due to the very small

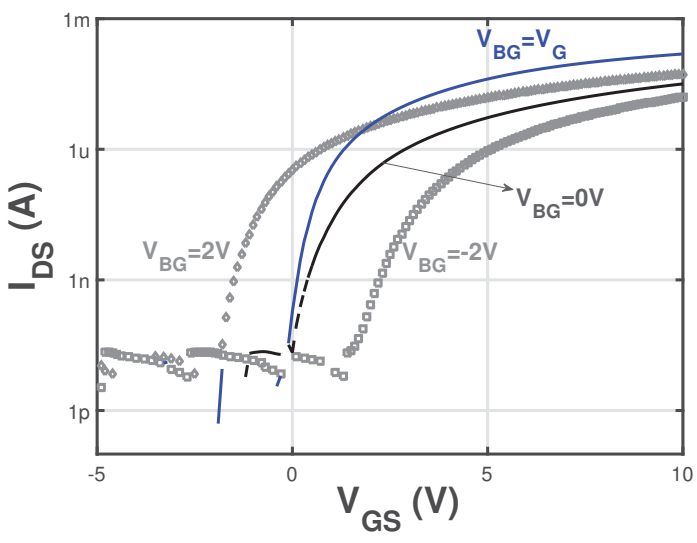

Fig. 5. Measured transfer characteristics of SADG IGZO TFT when back gate $(\mathrm{BG})$ connected to various biases $(-2,0,2 \mathrm{~V})$ including when shorted to front gate $(\mathrm{G})$.

mismatch $(<0.2 \%)$. Each capacitor of the $\mathrm{C}-2 \mathrm{C}$ networks is $5 \mathrm{pF}$. The access switches of the $\mathrm{C}-2 \mathrm{C}$ network are dual gate transistors with shorted BG to G. Shorting BG to G improves the subthreshold-slope of the transistor decreasing the leakage current when TFTs are switched off, as is shown in Fig.5. In addition, shorting $\mathrm{BG}$ to $\mathrm{G}$ improves the on resistance of the switch compared to all the other bias options.

Two different implementations of the comparator have been designed, whereby the minimum TFT size has been downscaled from $20 \mu \mathrm{m}$ to $5 \mu \mathrm{m}$ (Fig.6). The transient response of the comparator with open-loop offset cancellation is shown in Fig.7 for those two channel length designs. Auto-zeroing with output offset storage is used to cancel the observed offset of TFTs of more than $100 \mathrm{mV}$, necessary considering both mobility and threshold voltage mismatch (fig.3). Fig.7 depicts the response of the comparator for two different input signals: $\Delta V_{i n}=0 \mathrm{~V}$ and $\Delta V_{i n}=10 \mathrm{mV}$ operating at (a) $3 \mathrm{kHz}$ for $\mathrm{L}=20 \mu \mathrm{m}$ design and (b) at $10 \mathrm{kHz}$ for the $\mathrm{L}=5 \mu \mathrm{m}$ design. Offset cancellation is necessary to decrease the minimum detectable $V_{L S B}$ and increase the achieved resolution of the ADC minimizing non-uniformities. A microcontroller is used to control the ADC. Recently, TFT digital control circuits have been demonstrated successfully [2] with more than 1300 IGZO TFTs for a $5 b$ ADC on flexible substrate.

\section{EXPERIMENTAL RESULTS}

In Fig. 8 a micrograph of the fabricated TFT ADC on $15 \mu \mathrm{m}$ flexible polyimide foil is shown. The total footprint of the design measures $8.88 \mathrm{~mm}^{2}$. The two main block are labeled on the micrograph image. The comparator and C-2C network occupy the majority of the space. A total number of 91 SADG TFTs and 34 capacitors $(\mathrm{C}=5 \mathrm{pF}$ each) for the $\mathrm{C}-2 \mathrm{C}$ and 6 capacitors $(32 \mathrm{pF}$ each) for the auto-zero offset cancellation are used in the ADC. The switches are designed with a channel length of $\mathrm{L}=5 \mu \mathrm{m}$ and the minimum TFT channel length in the comparator is $\mathrm{L}=20 \mu \mathrm{m}$. The ADCs maximum clock of $2 \mathrm{kHz}$ is a consequence of the long channel length of the comparator. 


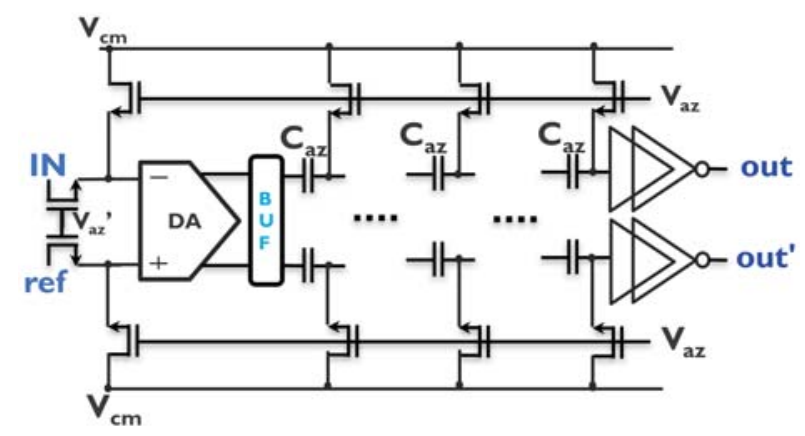

Fig. 6. The schematic of the comparator with offset cancellation and the voltage output buffers.

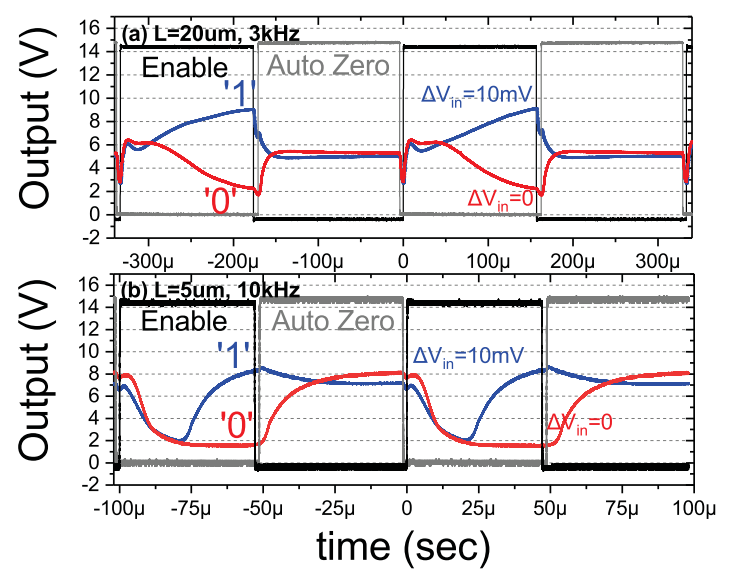

Fig. 7. Measured comparator output with auto-zero offset cancellation for designs using TFT of minimum (a) $\mathrm{L}=20 \mu \mathrm{m}$ at $3 \mathrm{kHz}$ and (b) $\mathrm{L}=5 \mu \mathrm{m}$ at $10 \mathrm{kHz}$.

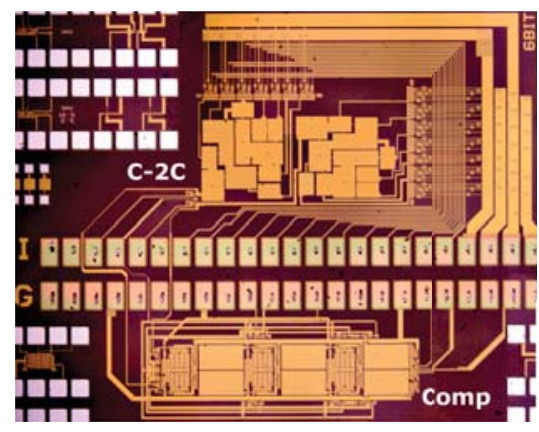

Fig. 8. A microphoto of the 6-bit MIM C-2C ADC on flexible substrate.

According to Fig.7 (b), sampling speed of the comparator can be increased to more than 5 times by scaling down the channel length to $5 \mu \mathrm{m}$, enabling a $1 \mathrm{fps}$ readout for a 1 megapixel fingerprint imager.

\section{EXPERIMENTAL RESULTS}

Fig. 9 shows the DNL and INL performance of the $\mathrm{L}=20 \mu \mathrm{m}$ $\mathrm{ADC}$ at $2 \mathrm{kHz}(134 \mathrm{~S} / \mathrm{s})$. The maximum obatined DNL and INL are $0.83 \mathrm{LSB}$ and 0.9LSB respectively at a clock frequency of

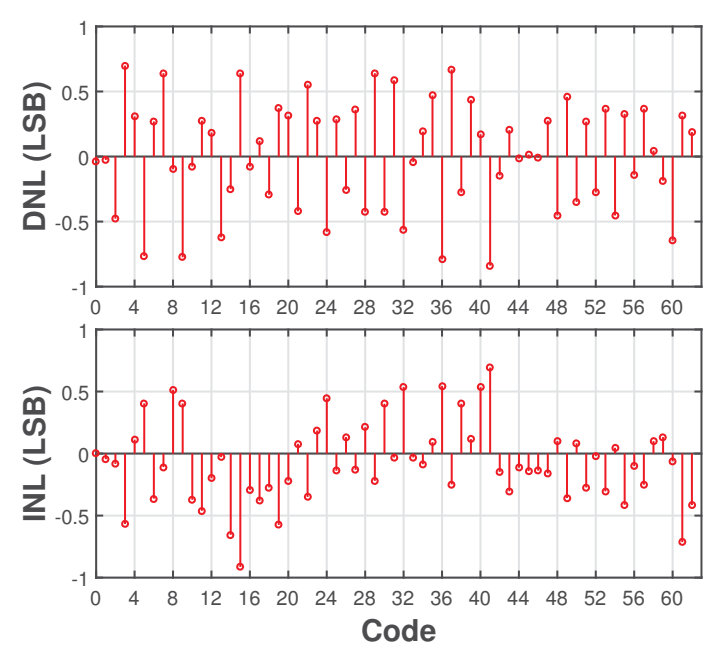

Fig. 9. Measured DNL and INL of the 6 bit MIM C-2C ADC operated at $2 \mathrm{kHz}$ on flexible substrate.

$2 \mathrm{kHz}$. The total power dissipation of the TFT-part of the ADC is $212 \mu \mathrm{W}$ at $V_{d d}=15 \mathrm{~V}$. whereby comparator dissipates only $6 \mu \mathrm{W}(2.8 \%)$. The majority of the power is dissipated by the 2-stage output buffers $(206 \mu \mathrm{W})$.

In Fig.10 the reconstructed sinusoidal wave signal (green circles) from the digital output of the ADC is shown compared to the $2.064 \mathrm{~Hz}$ sinusoidal wave (pink line) applied at the input of the ADC. In the bottom graph the FFT of the reconstructed sinusoidal wave signal is plotted to extract a $\mathrm{SNDR}=35.48 \mathrm{~dB}$. The calculated ENOB is 5.61 bit at a system clock of $2 \mathrm{kHz}$ $(134 \mathrm{~S} / \mathrm{s})$. The obtained figure of merit (FoM) of the ADC on foil with integrated offset cancellation is also therefore $16.58 \mathrm{~nJ} / \mathrm{c}$.s. for this $2 \mathrm{kHz}$ clock.

Table I displays a thin-film ADC state-of-the-art comparison and performance summary table. The presented TFT C-2C ADC on flexible substrate has $40 \%$ smaller FoM compared to state-of-the-art for unipolar technologies [2], [3], [10],[12] and is only $2 \mathrm{~nJ} / \mathrm{cs}$ larger than state-of-the-art of complementary TFT technologies. Moreover, the proposed ADC achieves DNL/INL figures smaller than 0.9LSB, demonstrating no missing codes. The proposed ADC has up to 5 times faster sampling speeds compared to earlier C-2C implementations and threefold smaller footprint. The larger power dissipation is mainly attributed to the high power dissipation of the output buffers.

\section{CONCLUSION}

This paper presents a 6 bit TFT-based C-2C ADC on thin flexible substrate $(15 \mu \mathrm{m})$ using SADG IGZO TFTs occupying only $8.88 \mathrm{~mm}^{2}$. The presented measurements achieve a SINAD $=35.48 \mathrm{~dB}$ and $\mathrm{ENOB}=5.61$ bit with $220 \mu \mathrm{W}$ dissipated power at $15 \mathrm{~V}$ supply. The ADC is operating at a clock up to $2 \mathrm{kHz}$ supplied externally from a microcontroller due to the $\mathrm{L}=20 \mu \mathrm{m}$ comparator. The calculated FoM is $16.58 \mathrm{~nJ} / \mathrm{c}$.s. Moreover, a more than 5 times faster comparator with downscaled SADG IGZO TFTs $(\mathrm{L}=5 \mu \mathrm{m})$ is demonstranted promis- 
TABLE I

PERFORMANCE SUMMARY AND STATE-OF-THE-ART COMPARISON TABLE (*CALCULATED)

\begin{tabular}{|c|c|c|c|c|c|c|c|c|c|}
\hline \multirow{3}{*}{$\begin{array}{c}\text { Technology } \\
\text { Architecture }\end{array}$} & \multicolumn{3}{|c|}{ Complementary Technology } & \multicolumn{6}{|c|}{ Unipolar Technology } \\
\hline & Poly-Si (2009) & Poly-Si (2010) & OTFT (2010) & a-Si:H (2012) & OTFT (2013) & \multicolumn{3}{|c|}{ DG ESL IGZO (2017-18) } & SADG IGZO \\
\hline & 2nd $\Sigma \Delta$ & Flash & SAR & Flash & VCO-based & \multicolumn{2}{|c|}{ ADSM } & C-2C SAR & C-2C SAR \\
\hline Tech Specs & $\begin{array}{c}\mathrm{L}=3 \mu \mathrm{m} 1 \mathrm{P} 2 \mathrm{M} \\
\text { LTPS }\end{array}$ & $\begin{array}{c}\mu_{n}=281 \mathrm{~cm}^{2} / \mathrm{Vs} \\
\mu_{p}=98 \mathrm{~cm}^{2} / \mathrm{Vs} \\
\mathrm{L}<8 \mu \mathrm{m}\end{array}$ & $\begin{array}{c}\mu_{n}=0.02 \mathrm{~cm}^{2} / \mathrm{Vs} \\
\mu_{p}=0.5 \mathrm{~cm} 2 / \mathrm{Vs} \\
\mathrm{L}=20 \mu \mathrm{m}\end{array}$ & $\begin{array}{c}\mathrm{BCE} \\
V_{T}=1.2 \mathrm{~V} \\
\mu_{n}=0.8 \mathrm{~cm}^{2} / \mathrm{Vs}\end{array}$ & $\mathrm{L}=5 \mu \mathrm{m}$ & \multicolumn{2}{|c|}{$\begin{array}{c}\mu_{n}=14 \mathrm{~cm}^{2} / \mathrm{Vs} \\
\mathrm{L}>15 \mu \mathrm{m}\end{array}$} & $\begin{array}{c}\mu_{n}=12.7 \mathrm{~cm}^{2} / \mathrm{Vs}, \\
V_{T}=3.7 \mathrm{~V}, \mathrm{~L}=30 \mu \mathrm{m}\end{array}$ & $\begin{array}{l}\mu_{E} F F=22.4 \mathrm{~cm}^{2} / \mathrm{Vs}, \\
V_{T}=2.56 \mathrm{~V}, \mathrm{~L}=20 \mu \mathrm{m}\end{array}$ \\
\hline ADC specs & $\begin{array}{c}\text { SDR }=69 \mathrm{~dB} \\
\text { SNDR }=65.63 \mathrm{~dB} \\
\text { OSR }=128\end{array}$ & 3 bit & 6 bit & 5 bit & $\begin{array}{c}\mathrm{SNR}=48 \mathrm{~dB} \\
\mathrm{ENOB}=7.7 \mathrm{bit}\end{array}$ & $\begin{array}{c}\mathrm{SNDR}=50 \mathrm{~dB} \\
\mathrm{ENOB}=8 \mathrm{bit}\end{array}$ & $\begin{array}{c}\text { SNDR=40dB } \\
\text { ENOB }=6 \mathrm{bit}\end{array}$ & $\begin{array}{c}\text { SNDR=35.9dB } \\
\text { ENOB }=5.7 \mathrm{bit}\end{array}$ & $\begin{array}{c}\mathrm{SNDR}=35.53 \mathrm{~dB} \\
\mathrm{ENOB}=5.61 \mathrm{bit}\end{array}$ \\
\hline DNL/ INL $(L S B)$ & - & $0.25 / 0.25$ & $-0.6 / 0.6$ & $1 / 1.8$ & $0.6 / 1$ & \multicolumn{2}{|c|}{ - } & $0.8 / 0.69$ & $0.83 / 0.9$ \\
\hline FoM (nJ/c.s.) & $14^{*}$ & - & - & - & 69 & 390 & 39 & 26 & 16.58 \\
\hline Power $(m W)$ & $63.3(11.2 \mathrm{~V})$ & $13 \mathrm{~V}$ & $0.004(3 \mathrm{~V})$ & $13.6(20 \mathrm{~V})$ & $0.048(20 \mathrm{~V})$ & \multicolumn{2}{|c|}{$2(20 \mathrm{~V})$} & $0.073(15 \mathrm{~V})$ & $0.212(15 \mathrm{~V})$ \\
\hline Sampling Rate $(S / s)$ & $400 \mathrm{k}$ & $3 \mathrm{M}$ & 10 & $2 \mathrm{k}$ & $167 \mathrm{~m}$ & 10 & 300 & 26.6 & 134 \\
\hline Footprint $\left(\mathrm{mm}^{2}\right)$ & 26 & 3.75 & 700 & - & 19.4 & \multicolumn{2}{|c|}{27.9} & 27.5 & 8.88 \\
\hline substrate & glass & Steel foil & glass & Flex compatible & foil & \multicolumn{2}{|c|}{ PI foil } & PI foil & PI foil \\
\hline Integration & Only analog & +Decoder & Only analog & Only analog & $\begin{array}{c}\text { +Metal resistors } \\
\text { +Logic }\end{array}$ & \multicolumn{2}{|c|}{ PWM output only } & $\begin{array}{c}\text { +offset cancellation } \\
\text { +DC biasing }\end{array}$ & +offset cancellation \\
\hline Authors & [8] & [9] & [10] & [11] & [12] & \multicolumn{2}{|c|}{ [3] } & [2] & This work \\
\hline
\end{tabular}
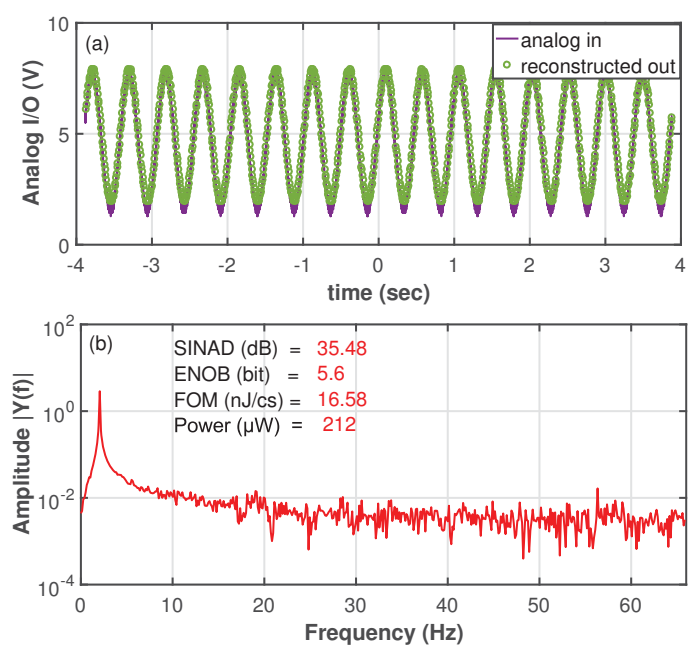

Fig. 10. (a) The analog input $2.061 \mathrm{~Hz}$ sinwave signal to the ADC and the reconstructed output points from the digital output of the ADC at a clock frequency of $2 \mathrm{kHz}$ and (b) the amplitude of the fft of the digitized output of the ADC.

ing faster ADCs aiming for in-panel fingerprint sensing for flat panel displays applications.

\section{ACKNOWLEDGMENT}

The authors thank the process engineers of Holst Centre's GEN1 Pilot Line for the realization of the ADCs. This work has received funding from the European Research Council (ERC) under the European Union's Horizon 2020 research and innovation program under grant agreement No 716426 (FLICs project) and Flexlines project within the Interreg Vprogramme Flanders-The Netherlands, a cross-border cooperation programme with financial support from the European Regional Development Fund, and co-financed by the Province of Noord-Brabant, The Netherlands.

\section{REFERENCES}

[1] C. Garripoli, et al. "A Fully Integrated $11.2 \mathrm{~mm}^{2}$ a-IGZO EMG Frontend Circuit on Flexible Substrate Achieving up to 41dB SNR and 29M Input Impedance," in IEEE Solid-State Circuits Letters. 2018

[2] N. P. Papadopoulos et al., Toward Temperature Tracking With Unipolar Metal-Oxide Thin-Film SAR C-2C ADC on Plastic, IEEE Journal of Solid-State Circuits, pp. 1-10, 2018.

[3] C. Garripoli, et al., 15.3 an a-igzo asynchronous delta-sigma modulator on foil achieving up to $43 \mathrm{db}$ snr and $40 \mathrm{db}$ sndr in $300 \mathrm{hz}$ bandwidth. In Solid-State Circuits Conference (ISSCC), 2017 IEEE International, pages 260-261. IEEE, 2017.

[4] K. Ishida, et al, "22.5 dB open-loop gain, $31 \mathrm{kHz}$ GBW pseudo-CMOS based operational amplifier with a-IGZO TFTs on a flexible film," SolidState Circuits Conference (A-SSCC), 2014 IEEE Asian, 2014, pp 313-316

[5] C. Zysset, et al, "IGZO TFT-Based All-Enhancement Operational Amplifier Bent to a Radius of $5 \mathrm{~mm}$," IEEE Electron Device Letters, vol. 34, no. 11, pp. 1394-1396, Nov. 2013.

[6] C. Garripoli et al., Analogue Frontend Amplifiers for Bio-Potential Measurements Manufactured With a-IGZO TFTs on Flexible Substrate, IEEE Journal on Emerging and Selected Topics in Circuits and Systems, vol. 7, no. 1, pp. 60-70, Mar. 2017.

[7] K. Myny, The development of flexible integrated circuits based on thinfilm transistors. In Nature Electronics, vol. 1, no.1, 2018, pg.30.

[8] W.-M. Lin, et al., A CBSC second-order sigma-delta modulator in $3 \mu \mathrm{m}$ LTPSTFT technology, in 2009 IEEE Asian Solid-State Circuits Conference (Taipei, 2009) pp. 133-136.

[9] A. Jamshidi-Roudbari, et al., A flash analog to digital converter on stainless steel foil substrate, Solid-State Electronics, Volume 54, Issue 4, April 2010, Pages 410-416.

[10] W. Xiong, et al., "A 3V $6 \mathrm{~b}$ successive-approximation ADC using complementary organic thin-film transistors on glass," 2010 IEEE International Solid-State Circuits Conference - (ISSCC), San Francisco, CA, 2010, pp. 134-135.

[11] A. Dey et al., "Amorphous silicon 5 bit flash analog to digital converter," Proceedings of the IEEE 2012 Custom Integrated Circuits Conference, San Jose, CA, 2012, pp. 1-4.

[12] D. Raiteri, et al, "An organic VCO-based ADC for quasi-static signals achieving 1LSB INL at 6b resolution," 2013 IEEE International SolidState Circuits Conference Digest of Technical Papers, San Francisco, CA, 2013, pp. 108-109.

[13] A. Kronemeijer, et al., "Dual-Gate Self-Aligned IGZO TFTs Monolithically Integrated with High-Temperature Bottom Moisture Barrier for Flexible AMOLED", SID Symposium Digest of Technical Papers, vol. 49, no. 1, pp. 1577-1580, 2018. 